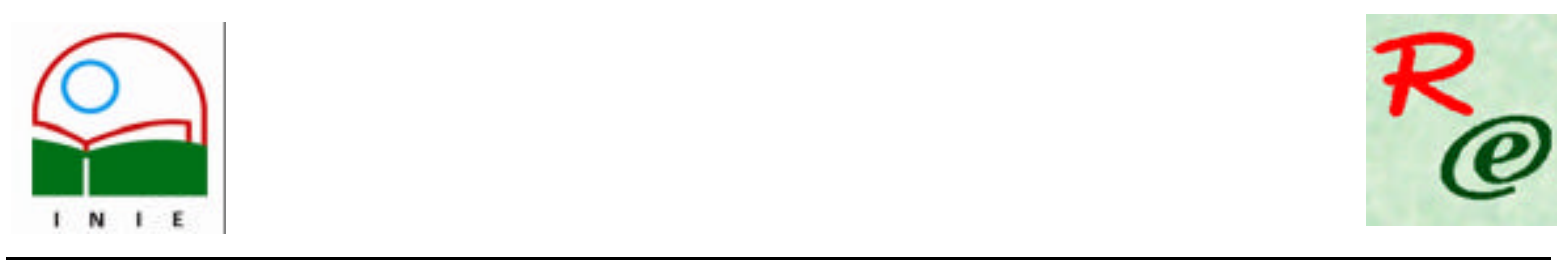

Actualidades Investigativas en Educación

Revista Electrónica publicada por el

Instituto de Investigación en Educación

Universidad de Costa Rica

ISSN 1409-4703

http://revista.inie.ucr.ac.cr

COSTA RICA

\title{
ENFOQUES TEÓRICOS Y DEFINICIONES DE LA TECNOLOGÍA EDUCATIVA EN EL SIGLO XX
}

THEORETICAL APPROACHES AND EXPLANATION OF EDUCATIONAL TECHNOLOGIES IN THE XX CENTURY

Volumen 9, Número 2

pp. $1-29$

Este número se publicó el 30 de agosto 2009

Manuel Luján Ferrer

Flora Salas Madriz

La revista está indexada en los directorios:

LATINDEX,$\underline{\text { REDALYC }}$ IRESIE, $\underline{\text { CLASE}}$ DIALNET, DOAJ, E-REVIST@S,

La revista está incluida en los sitios:

REDIE, RINACE, OEI, MAESTROTECA, PREAL, $\underline{\text { HUASCARAN, CLASCO }}$ 


\title{
ENFOQUES TEÓRICOS Y DEFINICIONES DE LA TECNOLOGÍA EDUCATIVA EN EL SIGLO XX
}

\author{
THEORETICAL APPROACHES AND EXPLANATION OF EDUCATIONAL TECHNOLOGIES \\ IN THE XX CENTURY
}

\author{
Manuel Luján Ferrer ${ }^{1}$ \\ Flora Salas Madriž
}

\begin{abstract}
Resumen: La tecnología educativa y los enfoques teóricos asociados al quehacer de los procesos de enseñanza y aprendizaje durante el siglo XX impactaron en la práctica educativa en las organizaciones de este tipo, la formación de los docentes, la selección, uso y evaluación de medios permitió valorar los aportes al sistema educativo sin descuidar las concepciones de las teorías de aprendizaje y la contribución de la didáctica al mejoramiento de la calidad. Este trabajo expone los principales enfoques teóricos y definiciones de la tecnología educativa en el siglo XX, su objetivo principal es presentar la evolución temática y las aplicaciones de la tecnología educativa y su relación con las diversas teorías de aprendizaje y la incorporación de los medios a la enseñanza.
\end{abstract}

Palabras clave: TECNOLOGÍA EDUCATIVA, INVESTIGACIÓN TECNOLOGÍAS PARA APRENDIZAJE, ENFOQUES TEÓRICOS Y MEDIOS PARA LA ENSEÑANZA Y EL APRENDIZAJE.

\begin{abstract}
The technology and the theoretical approaches associated to the task of the educational and learning processes during the $X X$ century impacted educative practices in the educative organiz ations, the formation of the education professionals, the selection, media use and evaluation allowed to value the contributions to the educative system without neglecting the conceptions of the theories of learning and the contribution of Didactics to the improvement of education quality. This work exposes the main theoretical approaches and definitions of the educative technology in the XX century; its primary target is to present/display the thematic evolution and the applications of the educative technology and their relation with the diverse theories of learning and the incorporation of media to education.
\end{abstract}

Key words: EDUCATIVE TECHNOLOGY, THEORETICAL AND AVERAGE INVESTIGATION TECHNOLOGIES FOR LEARNING, APPROACHES FOR EDUCATION AND THE LEARNING.

\footnotetext{
'Licenciado en Antropología Social (UCR), tiene una Maestría en Tecnología Educativa (Universidad Estatal a Distancia UNED, Costa Rica-Instituto Latinoamericano de Comunicación Educativa ILCE, México). Actualmente se desempeña como docente en la Escuela de Administración Educativa, en la Facultad de Educación de la Universidad de Costa Rica.

\section{Dirección electrónica:manulujan@gmail.com}

${ }^{2}$ Licenciada en Filosofía (UCR) y en Psicología (UCACIS), tiene una Maestría en Administración Universitaria (UCR) y es Candidata a Doctora en el Doctorado Latinoamericano en Educación de la Universidad de Costa Rica. Actualmente se desempeña como docente e investigadora en la Escuela de Administración Educativa, en la Facultad de Educación ce la Universidad de Costa Rica.
}

Dirección electrónica:flora.salas@ucr.ac.cr

Artículo recibido: 2 de febrero, 2009

Aprobado: 24 de julio, 2009 


\section{Introducción}

La investigación sobre las aplicaciones de las tecnologías de la información a la enseñanza se originó en los Estados Unidos, debido al potencial de esos medios para favorecer el adiestramiento y la capacitación militar, y porque era predecible que tuvieran usos en otros contextos, particularmente en el campo educativo.

Estados Unidos es el país que invierte más recursos en investigación educativa en el mundo, pese a que es limitada si se la compara con la que hace para la investigación en electrónica avanzada, desarrollo de productos farmacéuticos y en el campo militar. Se estima que la financiación de la investigación básica no es mayor al $0,1 \%$ sobre el $0.0001 \%$ del presupuesto total de educación (Casanova \& Berliner, 1997). Sin embargo, dadas las magnitudes del presupuesto de esa nación esta cifra, aparentemente pequeña, representa recursos importantes para el desarrollo de la investigación educativa. El nivel alcanzado en I+D en educación en ese país consolidó la comunidad más importante de investigadores, la American Educational Research Association (AERA), que es la asociación más grande de científicos dedicados a la investigación educativa en ese país. También, ello explica la influencia de esa nación en el desarrollo de las ciencias de la educación en todas las regiones del mundo, especialmente en aquellas donde la investigación y el desarrollo de conocimiento científico nuevo son escasos, como es el caso de América Latina.

Para la década de 1970, la informática y la tecnología educativa estuvieron entre los diez primeros temas tratados por la AERA. Pese a que en ese período la informática educativa fue una idea futurista, debido a que las computadoras eran muy costosas, el tema recibió buena acogida en la comunidad científica, desplazando a otros de interés en el pasado como: la enseñanza de la lengua, las matemáticas y la educación para los "desaventajados". Esto se explica debido a que la comunidad de especialistas e investigadores anticipó que la informática educativa marcaba un punto de inflexión en la historia reciente de la educación, que impactaría todas sus áreas y niveles.

Otro aspecto importante en la evolución teórica y la investigación sobre tecnologías para la enseñanza fue la ruptura conceptual creciente de muchos investigadores estadounidenses con la teoría conductista, que en los últimos veinticinco años implicó un paulatino acercamiento a nuevas teorías del aprendizaje de corte epistemológico cognitivo y constructivista, que se consolidaron en la década de 1990: 
El rápido aumento del interés por la psicología cognitiva refleja los cambios que están teniendo lugar en la psicología de la educación. Estos cambios se pueden relacionar, en parte, con la necesidad de estudiar los procesos que el conductismo desatendió: resolución de problemas, creatividad, etc. Pero también actuaron otros factores. La traducción de los trabajos de Piaget, y luego Vygotsky, tuvo un gran impacto en la investigación educativa en Estados Unidos. La influencia de estos investigadores, que no estaban atados por la tradición conductista del país, produjo un giro conceptual profundo en Estados Unidos. Hoy rigen en la cotidianidad las teorías constructivistas más que las conductistas. (Casanova y Berliner, 1997, p. 52)

Históricamente, las teorías del aprendizaje marcaron el desarrollo de la investigación educativa en los Estados Unidos. En la década de 1960, el gobierno decidió contratar a psicólogos experimentales para desarrollar proyectos educativos en el ejército e impulsar el aprendizaje de la ciencia en las escuelas, luego del lanzamiento del primer satélite soviético: el Sputnik en 1957. Este hecho científico y tecnológico sin precedentes en la conquista del espacio puso al descubierto la brecha que se abría entonces entre las dos potencias mundiales en el campo de la ciencia y la tecnología.

A inicios de la Guerra Fría el avance militar, la ciencia y la tecnología, eran estratégicos para el gobierno estadounidense. En ese período predominó el conductismo, cuyo máximo representante e investigador es B. F. Skinner, quien hizo importantes contribuciones al desarrollo educativo y militar de ese país en las décadas de 1940 y 1950.

Pese al adelanto de esa nación en el desarrollo de la ciencia y la tecnología, los problemas educativos se fueron haciendo más complejos en el marco de la revolución cultural de las décadas de 1960 y 1970. En ese período hubo asesinatos de importantes figuras políticas, conquistas trascendentales del movimiento por los derechos civiles, y crecieron el feminismo, los grupos en pro de la libertad de expresión y las protestas por la guerra de Vietnam (Casanova y Berliner, 1997).

En un nuevo contexto social y cultural la comunidad norteamericana de investigadores de la educación reconoció que los abordajes educativos tradicionales, cuya fundamentación teórico-pedagógica era el Conductismo, resultaban demasiado simplistas y limitaban el desarrollo de pensamiento de alto nivel en estudiantes y docentes. Esta crítica y revisión de las implicaciones educativas de la pedagogía conductista provocó un interés creciente por el 
estudio de problemas educativos con base en las teorías del aprendizaje de la psicología cognitiva y, posteriormente, en las décadas de 1980 y 1990, a partir de las concepciones sobre el aprendizaje de las psicologías psicogenética (Piaget) y sociocultural (Vygotski).

En relación con las tecnologías de la información, en la década de 1980 Papert, Novack, Ausubel, Minsky, Gowin, Hanesian, y Resnick, entre los más importantes, desarrollaron una nueva línea de investigación educativa en los Estados Unidos, que se fundamentó en las teorías cognitiva y psicogenética, y tendría un impacto importante en el estudio de esos medios y sus aplicaciones en la enseñanza. La investigación se centró en los procesos de instrucción y aprendizaje desde el aprendiz y la "mediación" de las tecnologías de la información. Ese proceso de cambio, aunado al abaratamiento de las computadoras, el desarrollo de software y a la revolución en las telecomunicaciones, particularmente de la Internet, favoreció que las tecnologías de la información y la comunicación digitales se posicionaran en un lugar privilegiado de la investigación educativa estadounidense.

En la década de 1990, los avances en investigación educativa en los Estados Unidos sobre las aplicaciones de las nuevas tecnologías digitales para apoyar e innovar el aprendizaje tuvieron impacto en Europa, donde para entonces, en el IV Programa marco de la Comisión Europea, se declararon prioritarias tres áreas de investigación en educación:

- Políticas educativas y formativas: estudios comparativos de políticas; adaptación al cambio; las respuestas de los sistemas educativos europeos a la diversidad cultural.

- Calidad e innovación en educación y formación: efectividad educativa; entornos de aprendizaje innovadores, en particular con respecto a la enseñanza de la ciencia y la tecnología; el impacto de las nuevas tecnologías en la educación y la formación.

- Educación, formación y desarrollo económico: evaluación de necesidades educativas y formativas; organizaciones y distribución de la información. (Calderhead, 1997, p. 12)

Al igual que en Europa fue a comienzos de la década de 1990 que se empezó a estudiar el impacto de las TIC en la educación en América Latina. 


\section{Los orígenes del concepto y líneas de investigación sobre tecnologías para el aprendizaje}

Los comienzos de la investigación empírica sobre los medios de enseñanza se remontan a la década de 1920, con los estudios de Thorndike y Gates sobre el recuento de palabras en textos escolares en los Estados Unidos. En esa misma década, el psicólogo estadounidense Sidney Leavitt Pressey diseñó las primeras máquinas para apoyar la enseñanza, creadas para dar una respuesta correcta en forma inmediata al estudiante en pruebas de elección múltiple, para que los errores sirvieran para mejorar el desempeño en las respuestas.

El principio pedagógico de Leavitt fue crear las condiciones para el reforzamiento individual de los aprendizajes, porque el estudiante tenía la oportunidad de repetir los ejercicios tantas veces como fuera necesario. Esas máquinas condujeron a la enseñanza programada, entendida como un método pedagógico en el que se transmiten conocimientos sin la mediación de un docente o tutor, en el que las prácticas de enseñanza se ajustan a las características personales del aprendiz, quien tiene el control sobre la ejecución y el avance de las actividades de aprendizaje. Conforme se extendieron experiencias de ese tipo se incrementó la investigación sobre la enseñanza programada, la televisión educativa y el cine didáctico. Para entonces, aún no se había acuñado el término "tecnología educativa" (Gallego, 1997).

Para Chadwick (Bartolomé, 2001), el concepto de "tecnología educativa" empezó a usarse en los Estados Unidos, en la década de 1960; sin embargo, ya se utilizaba después de la Segunda Guerra Mundial en un sentido amplio cuando se empleaba el término para hacer referencia a los medios de comunicación aplicados en educación. Debido a la influencia de las investigaciones sobre el análisis y modificación de conducta se definió la "tecnología educativa" como diseño de estrategias, uso de medios y control de sistemas de comunicación para la enseñanza. Las primeras influencias para el desarrollo de la tecnología educativa como campo de investigación fueron:

1. La existencia de un interés por las diferencias individuales del aprendizaje en la década de los años 50, citando la investigación educativa militar, en el desarrollo de aparatos para la autoenseñanza, los programas ramificados de Crowder (1959), y aplicaciones de la computadora a la enseñanza. 
2. La ciencia conductual y su teoría del aprendizaje, que tiene como referencia anterior la teoría de contigüidad de Guthrie (1935) y como principal exponente a Skinner.

3. La tecnología representada por los modernos equipos de cine, televisión y vídeo tape. A finales de la década de los 50, Ampex produce el primer sistema de registro magnético de la señal de vídeo. (Bartolomé, 2001, p.2)

El concepto de tecnología educativa emergió vinculado al uso en educación de los medios audiovisuales modernos y así fue recogido por la UNESCO en 1984.

B. F. Skinner (1904-1990), uno de los máximos representantes e investigadores de la tecnología educativa en sus comienzos, planteó en 1954 la posibilidad de la "tecnificación de la enseñanza" mediante el uso de máquinas en su libro The Science of Learning and de Art of Teaching. Ese libro pasó casi inadvertido, pero el siguiente, Teaching Machines, fue recibido con mayor interés por la comunidad de investigadores de la tecnología aplicada a la educación.

Como se puede observar, el origen del campo de investigación en tecnología educativa se asocia a eventos históricos, intereses político-militares, a la psicología del aprendizaje, al enfoque sistemático y al desarrollo de los medios de comunicación. De igual forma, se le vincula con la teoría de la comunicación y la información, y sus aplicaciones en el campo educativo:

Uno de los fenómenos característicos del siglo XX es el gran desarrollo de los medios de comunicación. Y concomitante con la aparición de métodos, aparatos y dispositivos para transmitir y procesar textos, cifras, sonido e imágenes, se ha desarrollado una teoría unificadora denominada teoría de la información, la cual es sujeto y objeto de interés prioritario para estudiosos e investigadores. La teoría de la información es una representación matemática de los elementos, las condiciones y los parámetros que comprenden la transmisión y el procesamiento de información. Se deriva de los planteamientos originales de Claude E. Shannon sobre la teoría matemática de la comunicación, publicados en 1945 y 1948. (Patiño, 2002, p. 33) 
La investigación en tecnología educativa tuvo sus orígenes dentro Pragmatismo de James y el Conductismo de Skynner, pero a partir de la década de 1980 se fundamentó también en la teoría cognitiva, por lo que hay una evolución de la investigación sobre medios del enfoque del aprendizaje del conductismo al de la psicología cognitiva (Bartolomé, 2001; Cabero, 2001; Gallego, 1997). En esa evolución hay "momentos" o "enfoques" en la investigación, que son:

1. El Enfoque de los medios: la tecnología se identifica inicialmente con los medios.

2. El Enfoque conductista: la tecnología es asociada al análisis y modificaciones de la conducta.

3. El Enfoque sistémico: la tecnología es concebida como parte de un proceso de aprendizaje global y de coordinación de variables, con especial énfasis en los elementos cibernéticos.

4. El Enfoque ecológico: estudia el conjunto de relaciones interpersonales que confluyen en el sistema educativo mediado por tecnología, de forma que el diseño de la misma no es concebido como una estructura o sistema de relaciones estáticas; sino más bien, se le visualiza como una red fluyente, consecuencia de la presencia o aparición de elementos imprevistos en la interacción entre el aprendiz, el docente y el medio. (Bartolomé, 2001, pp. 4-5)

En el desarrollo de la investigación educativa, los "medios" no se han conceptualizado de igual forma. En el cuadro 1 se hace una síntesis cronológica de las principales concepciones de la tecnología educativa en los últimos cincuenta años, que incluye 45 definiciones diferentes, en las que se señalan por su importancia y aportes la de Papert, la de la Association for Educational Communications and Technology de los Estados Unidos y en el contexto iberoamericano la postura de Julio Cabero. 


\section{Cuadro 1}

Concepción de los medios para la enseñanza según distintos investigadores de la educación, por autor y año

\section{Definiciones de tecnología educativa (1940-1999)}

1.B. F. Skinner (1904-1990). La enseñanza programada es un método de enseñanza sin mediación de un profesor o tutor en el que se pueden emplear máquinas, computadoras, libros, o cualquier otro recurso didáctico que permita que el estudiante trabaje en forma independiente y aprenda a su propio ritmo.

2. ElY (1963): La tecnología educacional es aquel campo de la teoría y práctica educativa, involucrada principalmente con el diseño y uso de mensajes que controlan el proceso de aprendizaje.

3. MEREDITH (1965): "... un medio educativo no es meramente un material o un instrumento, sino una organización de recursos que media la acción entre maestro y alumno".

4. GAGNE R. M. (1968): La tecnología educativa es un cuerpo de conocimientos técnicos con relación al diseño sistémico y la conducción en la educación, con base en la investigación científica.

5. KOMOSKY (1969): Lo que el tecnólogo educativo sabe es que su función crucial como educador radica en que estructura el medio ambiente en favor de la educación y que este proceso de estructuración educacional o instruccional del medio es la tecnología a través de la cual aspectos reproducibles del arte de educar, pueden ser analizados efectivamente así como imitados.

6. COLLIER et. al. (1969): La tecnología educacional, en el sentido más amplio, abarca la aplicación de sistemas, técnicas y materiales para mejorar el proceso del aprendizaje.

7. TICKTON, S. (1970): Un modo sistemático de diseñar, operar y evaluar el proceso total de aprendizaje y enseñanza en función de objetivos específicos, basado en la investigación del aprendizaje y la comunicación humanos, que emplea una combinación de recursos humanos y no humanos para lograr una instrucción más efectiva.

8. ROSSI y BIDLLE (1970): "... un medio es cualquier dispositivo o equipo que se usa normalmente para transmitir información entre las personas [...]. Un medio educativo es un dispositivo de este tipo que se utiliza con fines educativos" (perspectiva instrumental y finalidad comunicativa o informativa).

9. GASS (1971): Es la concepción orgánica y la puesta en práctica de sistemas de aprendizaje que utilizan provechosamente los sistemas modernos de comunicación, los materiales visuales, la organización de la clase y los métodos de enseñanza sin esperar milagros de ellos.

10. OFESH (1971): La tecnología educativa es la aplicación sistemática de los conocimientos científicos a la solución de problemas educacionales. 


\section{Definiciones de tecnología educativa (1940-1999)}

11. DAVIS (1971): Considera que la tecnología educacional presenta dos aspectos: uno referente a los equipos (hardware) y otros a los programas (software).

12. Agencia Norteamericana para el Desarrollo Internacional (1972): La tecnología educativa es una forma sistemática de planificar, implementar y evaluar el proceso total de aprendizaje y de la instrucción en términos de problemas específicos basados en las investigaciones humanas, empleando una combinación de recursos y materiales con el objeto de obtener una instrucción más efectiva.

13. OLSON y BRUNER (1974): el aprendizaje se puede realizar a través de la experiencia directa o de una experiencia vicaria o mediadora.

14. SALOMON (1974), concibe los medios como el resultado de la interacción de tres elementos: el sistema simbólico, el mensaje y la tecnología de transmisión.

15. GAGNE (1975): La tecnología educativa es un conjunto de técnicas sistemáticas acompañadas de un conocimiento práctico, puesto al servicio de la planificación, control y operación de escuelas, vistas como sistemas educacionales.

16. Asociación para la Comunicación y Tecnología Educacional (AECT) (1977): La tecnología educativa es un proceso complejo e integrado que incluye personas, procedimientos, ideas, aparatos y organizaciones para analizar problemas y proyectar, aplicar, evaluar y administrar soluciones a estos problemas relacionados con todos los aspectos del aprendizaje humano.

17. MITCHEL (1977): La tecnología educativa es el área de estudio y de práctica (en educación) que se ocupa de todos los aspectos de organizaciones de los sistemas y procedimientos educativos y busca asignar los recursos para la obtención de resultados educacionales específicos y potencialmente repetibles.

18. SCHRAMM (1977) "los medios son formas o vehículos replicables a través de los que se da forma, se almacena y se entrega la instrucción al estudiante".

19. HEIDT (1978): "Software con el necesario hardware, en un contexto particular de comunicación instructiva".

20. Centro de Experimentación para el Desarrollo de la Formación Tecnológica (CEDEFT) (1978): Tecnología educativa es la aplicación sistemática del conocimiento científico y organizado a la solución de problemas, con el propósito de lograr la eficiencia y la efectividad del sistema educativo.

21. CASTANEDA (1978): "... un objeto, un recurso instruccional que proporciona al alumno una experiencia indirecta de la realidad, y que implica tanto la organización didáctica del mensaje que se desea comunicar como el equipo técnico necesario para materializar este mensaje".

22. Organización de Estados Americanos (OEA) (1978): La tecnología educativa se entiende como la aplicación de un proceso metódico, diseñado para enfrentar y resolver problemas en el sistema educativo con el objetivo de hacer óptima su operación. 


\section{Definiciones de tecnología educativa (1940-1999)}

23. CHADWICK, C. (1978): La aplicación de un enfoque científico y sistemático con la información concomitante al mejoramiento de la educación en sus variadas manifestaciones y niveles diversos.

24. GERLACH y ELY (1979): "... es cualquier persona, material o acontecimiento que establece las condiciones para que el alumno adquiera conocimientos, capacidades y actitudes".

25. WOOD, A. (1979): La tecnología educativa como la aplicación sistemática del conocimiento organizado a las tareas prácticas de la educación, y al técnico educacional como a la persona que trata de comprender la teoría y de aplicarlo.

26. CONTRERAS OGALDE (1980): La tecnología educativa es la aplicación de conocimientos científicos a la solución de problemas de la educación. "La Tecnología Educativa aborda el proceso enseñanza-aprendizaje en forma sistemática y organizada y nos proporciona estrategias, procedimientos y medios emanados de los conocimientos científicos en que se sustenta".

27. UNESCO (1980): La tecnología educativa es una aplicación sistemática de los recursos del conocimiento científico al proceso que necesita cada individuo para adquirir y utilizar conocimientos.

28. PENALOZA, W. (1980): Conjunto de procedimientos, técnicas e instrumentos que se emplean para la plasmación en los hechos de una concepción educacional. Es por lo tanto, algo más que la aplicación de los principios de la Ciencias para resolver los problemas de la educación.

29. 1er. Congreso Nacional de Investigación Educativa (México) (1981): La tecnología educativa es un conjunto de procedimientos o métodos, técnicas, instrumentos y medios derivados del conocimiento, organizados sistemáticamente en un proceso, para obtener productos o resultados educativos de manera eficaz y replicable.

30. ESCUDERO (1983): "... cualquier objeto o recurso tecnológico (con ello se alude a su soporte físico) que articula en un determinado sistema de símbolos ciertos mensajes (el contenido) en orden a su funcionamiento en contextos instructivos (el enfoque)".

31. CHADWICK (1986): Tecnología Educativa es el enfoque sistemático y la aplicación de un conjunto de conocimientos científicos para el ordenamiento del ámbito específico conocido con el nombre de educación.

32. UNESCO (1986). Aplicación de todo sistema, técnica o material que permite mejorar el proceso de enseñanzaaprendizaje, tomando en cuenta tanto los recursos técnicos como los humanos y su interacción con el fin de conseguir la mayor eficacia posible. En este sentido, la tecnología de la educación emplea el análisis de sistemas como instrumento teórico.

33. FERNANDEZ, SARRAMONA y TARIN (1988): "... soportes materiales en los cuales se presentan contenidos y sobre los cuales se realizan las distintas actividades", distinguiéndolos así de los métodos. 


\section{Definiciones de tecnología educativa (1940-1999)}

34. SEVILLANO (1990), destacando las relaciones con el contexto, los considera elementos configuradores de una nueva relación entre profesor-alumno, aula, medio ambiente, contenido... que incide en los procesos cognitivos y actitudinales de los alumnos, transformando incluso los mismos roles de las instituciones docentes.

35. VILLARROEL C. (1990): Conjunto de técnicas y procedimientos concebidos por la Ciencia de la Educación para resolver problemas educativos y/o educacionales.

36. QUESADA R. (1990): Estudio científico de las reglas de procedimientos que persiguen modificar una práctica educativa.

37. Dirección de Investigación y Comunicación Educativa (ILCE) (1993): Modelo científicotecnológico en educación.

38. BRAVO C. (1993): La Tecnología Educativa es la aplicación de manera creadora de las técnicas y procedimientos para el mejoramiento del sistema educativo y para la prevención y solución de los problemas en la que juega un papel importante el enfoque sistémico, la eficiencia en la gestión y dirección educativa, la selección adecuada de los medios de enseñanza y las investigaciones en el área pedagógica.

39. LITWIN, E. (1994): El desarrollo de propuestas de acción basadas en disciplinas científicas referidas a las prácticas de enseñanza que, incorporando todos los medios a su alcance, dan cuenta de los fines de la educación en los contextos sociohistóricos que le otorgan significación.

40. FERNANDEZ B. y PARRA I. (1995): Concepción pedagógica innovadora que en cualquier nivel de enseñanza se realice con el propósito de transformar al ser humano y su realidad social.

41. PAPERT (1995): los medios son herramientas que permiten la construcción del conocimiento. Particularmente, la computadora hace posible que el aprendiz fabrique cosas "tangibles", fuera de la mente, que tienen significado para nosotros como personas. Esa es la tesis central del "construccionismo".

42. ALONSO (1996) entiende los medios de enseñanza como "los instrumentos, equipos o materiales, concebidos como elementos curriculares mediadores de la experiencia directa, que articulan en un determinado sistema de símbolos ciertos mensajes y persiguen la optimización del proceso de enseñanza y aprendizaje".

43. En 1996, la Association for Educational Communications and Technology de los Estados Unidos, define tecnología educativa como: “... a complex, integrated process involving people, procedures, ideas, devices, and organization for analyzing problems, and devising, implementing, evaluating, and managing solutions to these problems, in situations in which learning is purposive and controlled".

44. GOMEZ M. (1997): La Tecnología Educativa es un arte aplicada, capaz de favorecer en la comunidad escolar la movilización de la información, el surgimiento y desarrollo de potencialidades individuales y colectivas, la participación crítica, constructiva y responsable dentro de una visión socio-cultural. 


\section{Definiciones de tecnología educativa (1940-1999)}

45. CABERO (1999) los define como "los elementos curriculares que, por sus sistemas simbólicos y estrategias de utilización propician el desarrollo de habilidades cognitivas en los sujetos, en un contexto determinado, facilitando y estimulando la intervención mediada sobre la realidad, la captación y comprensión de la información por el alumno y la creación de entornos diferenciados que propicien los aprendizajes".

Fuentes: (Cabero, 2001; Kozma, 2003; Marquès, 1999; Papert, 1995; Verdecia, 2007).

El cuadro anterior muestra que el campo de la tecnología educativa pasó en la segunda década del siglo XX por un proceso de transición de una fase pre-científica y especulativa, a una más apegada a los requerimientos de la investigación científica. El hecho de que cada autor u organización emitiera una definición "propia" de la tecnología educativa evidencia que su fundamentación era más ideológico-política que científica. Esta es una característica propia de las áreas del conocimiento que no alcanzan el consenso necesario para constituir comunidades científicas y, en consecuencia, paradigmas (Kuhn, 1980).

Debido a la variedad de concepciones de los medios, así como a los diferentes enfoques teóricos se debe considerar que los problemas, temáticas y ámbitos de investigación en las tecnologías aplicadas a la educación han sido y serán variados, pues surgen de ideas igualmente diversas sobre el campo mismo de estudio (Gallego, 1997). Por esa razón, en la actualidad se cuenta con varias clasificaciones de la investigación en tecnología educativa, con diferentes perspectivas epistemológicas, teóricas, metodológicas y técnicas.

\section{Evolución histórica de la investigación y debates sobre medios y tecnología educativa}

Se pueden distinguir las siguientes tendencias de investigación en el campo de los medios, los materiales de enseñanza y las nuevas tecnologías (Cabero, 2001, p. 473):

1. Semiológica: donde se analizan los diferentes sistemas simbólicos movilizados en los medios y sus cargas expresivas, estéticas y comunicativas.

2. Marxista: donde se analizan aspectos como la alienación, el conflicto de clase y, en general, la sociedad consumista que se desarrolla y propicia con los medios que Volumen 9, Número 1, Año 2009, ISSN 1409-4703 
permiten su mantenimiento. Además, interesa conocer su repercusión en los ámbitos político, económico, social y cultural.

3. Psicoanalista: en la cual se reflexiona sobre las aportaciones psicológicas que las personas incorporamos a los medios, como consecuencia de nuestra personalidad y cómo se produce una unión medio-persona en un espacio vivencial que supera lo lúdico y la diversión.

4. Sociológica: en la que se analiza el impacto de los medios como elementos culturales y formadores de pautas y aprendizaje de las conductas de la sociedad en la que vivimos y nos desarrollamos.

5. Didáctico-curricular: donde se estudia el papel que los medios pueden jugar en los contextos de formación, y las inquietudes específicas que en ellos se originan en cuanto al diseño de sus mensajes, la adecuación a los receptores, y su explotación en el ámbito educativo.

Distintos campos teóricos han servido de "telón de fondo" para analizar algunas implicaciones de la tecnología educativa en distintos escenarios relacionados con la sociedad y el sujeto. En este sentido, el Marxismo y el Psicoanálisis han investigado esas tecnologías con perspectivas muy distintas. El primero como medios de "alienación"; el segundo, como medios que se integran a los procesos psíquicos primarios, en particular los de naturaleza inconsciente. Pese a los aportes de estas investigaciones, no se han enfocado en sus implicaciones en los procesos de enseñanza y aprendizaje, sino en consecuencias no deseadas y no previstas de los medios de comunicación de masas para la sociedad y el sujeto.

La investigación también se ha clasificado a partir de la posición teórica que la apoya; por eso, Clark y Sugrue (Cabero, 2001, p. 479), proponen los siguientes enfoques: 


\section{Cuadro 2}

Enfoques de investigación en medios de enseñanza, según sus referentes teóricos

\begin{tabular}{|c|c|}
\hline $\begin{array}{l}\text { Tipo de } \\
\text { investigación/ } \\
\text { Enfoque teórico }\end{array}$ & Cuestiones de investigación \\
\hline Conductista & - Efectos del tipo de medios sobre el rendimiento. \\
\hline Cognitivas & $\begin{array}{l}\text { - Efectos de los atributos de los medios sobre el procesamiento } \\
\text { cognitivo y/o el rendimiento. } \\
\text { - Efectos del método instruccional sobre el procesamiento cognitivo y el } \\
\text { rendimiento. } \\
\text { - Efectos de la interacción de las aptitudes del estudiante y el método } \\
\text { instruccional sobre el procesamiento cognitivo y el rendimiento. }\end{array}$ \\
\hline $\begin{array}{l}\text { Cuestiones } \\
\text { actitudinales }\end{array}$ & $\begin{array}{l}\text { - Efectos de la interacción de las actitudes/atribuciones/expectativas de } \\
\text { los estudiantes y del método instruccional o del medio sobre el } \\
\text { procesamiento cognitivo y el rendimiento. }\end{array}$ \\
\hline $\begin{array}{l}\text { Cuestiones } \\
\text { económicas }\end{array}$ & $\begin{array}{l}\text { - Efecto del medio sobre el coste de la instrucción. } \\
\text { - Efecto del medio sobre el tiempo en la instrucción. }\end{array}$ \\
\hline
\end{tabular}

Fuente: Cabero (2001)

Los enfoques Conductista, Cognitivo y Funcionalista de investigación del cuadro anterior, indican que los estudios relacionados con la tecnología aplicada a la educación se centran en aplicaciones concretas de esos medios en el campo educativo. Como es evidente, los problemas de investigación se corresponden a los enfoques teóricos particulares, según su concepción propia del aprendzaje y, en consecuencia, de la enseñanza y la mediación pedagógica. Interesa resaltar el énfasis en el rendimiento, eficiencia y eficacia de los medios, que confirma una perspectiva pragmática de la educación, que no va más allá de los efectos observables y cuantificables de los procesos de enseñanza. En este sentido, la investigación fue principalmente de tipo positivista, privilegiando estudios experimentales y cuasi-experimentales.

En el cuadro 3, se desglosan líneas de investigación que han predominado en los estudios sobre medios (Gallego, 1997). En ellas aparecen tendencias innovadoras, porque se plantean problemas de investigación que trascienden los "efectos observables" de la tecnología aplicada a la enseñanza y el aprendizaje, al abordar las implicaciones de los medios para quienes los utilizan: docentes y estudiantes. Esto marca un punto de inflexión 
entre las teorías y enfoques de investigación positivista y funcionalista, y los de corte fenomenológico, crítico y sistémico-complejo.

\section{Cuadro 3}

Líneas de investigación en medios, según Gallego (1997)

\begin{tabular}{|c|c|}
\hline Enfoque & Líneas de investigación \\
\hline $\begin{array}{l}\text { El estudio del medio en sí } \\
\text { mismo }\end{array}$ & $\begin{array}{l}\text { Diseño y evaluación de programas (software, } \\
\text { hardware y courseware). }\end{array}$ \\
\hline $\begin{array}{l}\text { El estudio del aprendizaje } \\
\text { con medios }\end{array}$ & $\begin{array}{l}\text { Investigación desde el enfoque cognitivo. } \\
\text { Estudios sobre motivación, actitudes y expectativas } \\
\text { de los alumnos. }\end{array}$ \\
\hline $\begin{array}{l}\text { El enfoque didáctico- } \\
\text { curricular y el análisis de los } \\
\text { medios desde la } \\
\text { perspectiva del profesor }\end{array}$ & $\begin{array}{l}\text { Cuestiones actitudinales: opiniones y valoraciones. } \\
\text { - Cuestiones prácticas: decisiones sobre selección, uso } \\
\text { - Cuestiones formativas: formación inicial/en servicio. } \\
\text { - Cuestiones organizativas: contextos de aplicación. }\end{array}$ \\
\hline
\end{tabular}

Fuente: Gallego (1997)

En particular, el enfoque didáctico-curricular hizo aportes importantes a la didáctica, porque la investigación se centró en contextos naturales de desarrollo curricular de los medios, tomando en cuenta los siguientes aspectos:

1. Cuestiones actitudinales, referentes al análisis de opiniones, valoraciones y percepciones de los medios por parte de los(as) profesores(as) (Chandra, 1987, Hativa, Shapira y Navon, 1990, Gallego y Camacho, 1991, Castaño, 1992, Delcourt y Kinzie, 1993, Gallego, 1994c y d).

2. Cuestiones prácticas, centradas en las decisiones sobre selección, uso y evaluación de medios por parte de los(as) profesores(as), y en el conocimiento y acción de los(as) profesores(as) con/sobre medios (Olson, 1986, 1988, Area, 1991, Cabero, 1992, Veen, 1993, Gallego, 1994f). 
3. Cuestiones formativas, trazadas en torno al análisis de estrategias de formación y desarrollo profesional del profesorado en medios de enseñanza, tanto en formación inicial como permanente, a través de procesos colaborativos de elaboración de materiales curriculares o de introducción de nuevos medios en los centros (Montero y Vez, 1992, Gallego, 1994a y b, 1995, Cebrián, 1993).

4. Cuestiones organizativas, dirigidas al análisis del impacto de los medios en la organización de la escuela, reorganizando las interacciones y el ambiente físico en el aula (Pitt, 1993) e influyendo en los contextos de aplicación (Plomp y Pelgrum, 1992), a través de novedades como las redes de comunicación que posibiliten no sólo inéditas formas organizativas sino también la colaboración y el desarrollo profesional (Guskey, 1994, DiMauro y Jacobs, 1995). (Gallego, 1997)

La investigación en medios de enseñanza también ha seguido las mismas líneas paradigmáticas de la didáctica, por lo que pasó del modelo proceso-producto, al mediacional y, finalmente, al etnográfico (Cabero, 1991).

La superación de una visión del uso de los medios en la enseñanza reduccionistapositivista por una compleja-cualitativa ha sido lenta. Hasta finales de la década de 1980 predominó una concepción "fisicalista" salida de la tradición conductista, que considera que la utilización didáctica de la tecnología se limita al conocimiento y manipulación instrumentales. Manipular "botones" es lo único que debe saber el docente sobre el medio, lo que provocó el retraso en la utilización no tradicional en el aula de los nuevos dispositivos digitales, específicamente en el caso de la computadora (Cabero, 2001; Gallego, 1997).

En la década de 1990 por primera vez se llevaron a cabo estudios sobre la formación docente en el uso didáctico de medios:

... en la mayoría [de los planes de estudio para la formación docente] suele existir una ausencia en la formación en estos elementos didácticos, contradictorio con la limitada formación que los(as) profesores(as) indican poseer en el dominio técnico, sémico y didáctico de los medios y con las veces que tal formación ha sido reclamada (Gimeno Sacristán y Fernández Pérez, 1980; ITE, 1985; López-Arenas y otros, 1988; Cabero, 1988ª). En este aspecto de la formación del profesorado sería bueno pensar sobre cuántos planes institucionales de introducción de medios en la escuela, comienzan por formar al profesorado "antes de" o "paralelamente a", e incluso "después de", la presencia física de los medios en los centros. (Cabero, 1991, p. 2) 
Otro problema que hasta ahora obstaculiza el avance sobre la visión "fisicalista" es de orden comercial, debido a que muchas empresas que producen medios buscan posicionar sus productos en el mercado, al margen de su utilidad real para la enseñanza y el aprendizaje. Esto sucedió con el libro de texto, la TV, el cine, el vídeo y las computadoras. La pregunta de investigación que orienta ese enfoque es: ¿el nuevo medio produce más rendimiento de aprendizaje que el medio antiguo o la instrucción convencional?

[AI respecto], como se afirma en diferentes meta-análisis sobre investigaciones en medios (Levie y Dickie, 1973; Clark, 1975, 1983, 1984 y 1989; Clark y Salomon, 1986; Escudero, 1983a y b; Moore y otros, 1986; Clark y Sugrue, 1988; Cabero, 1989; Salomon y Clark, 1977), el volumen de estudios y conclusiones, parece no relacionarse con un incremento en justificaciones para la inserción y utilización curricular de los medios. (Cabero, 1991, p. 2)

Como consecuencia del predominio de la concepción "fisicalista" de los medios de enseñanza, donde prevalecen estudios sobre las características del dispositivo, se ha ignorado el papel del profesorado y del estudiantado en el aprendizaje mediado por tecnologías. Ese "olvido" ha tenido consecuencias no deseadas y no previstas, y los resultados esperados a partir de grandes inversiones en equipo no se han dado, como resultado del:

... olvido de los investigadores del papel desempeñado por el profesor, el alumno, los contenidos, las estrategias de enseñanza utilizadas y el contexto; contemplando solamente como variables determinantes de los productos cognitivos y de rendimiento a alcanzar con un medio, sus características tecnológicas e instrumentales.

$[\ldots]$

Kulik y otros (1980) en un meta-análisis sobre investigaciones de medios, pusieron de manifiesto cómo los efectos positivos de un medio sobre otro se atenúan, cuando es un mismo profesor el que los utiliza en los diversos grupos experimentales constituidos. Clark (1984), tras realizar una revisión de las investigaciones en medios, llega entre otras a la conclusión de que es necesario seguir investigando en relación con las tareas de aprendizaje, los contenidos y el contexto curricular donde el medio se inserta. (Cabero, 1991, p. 3) 
Salomón y Clark, destacados investigadores estadounidenses en el campo de la tecnología educativa, en un artículo paradigmático sobre el tema titulado Reexamining the methodology of research on media and technology in education, publicado en 1977, distinguen dos líneas de indagación a las que denominaron "investigación con medios" e "investigación sobre medios". La primera busca el medio más eficaz para el proceso de enseñanza y aprendizaje, por lo que su interés se centra en el mecanismo de entrega de información y no en "cómo y a quién se le entrega". Las preguntas que orientan esos estudios son: ¿es el medio $A$ mejor que el medio $B$ ? o, ¿es la técnica $X$ más efectiva sobre el medio que la técnica $Y$ ? La segunda, se interesa por el análisis interno del proceso de enseñanza y aprendizaje mediado con tecnologías y estudia los sistemas simbólicos, los atributos y la estructuración de los contenidos, y cómo se asimilan para desarrollar habilidades cognitivas en los aprendices.

Luego de la revisión de la investigación en medios efectuada en los años setenta y ochenta por Clark y Salomón, concluyeron que los resultados logrados hasta entonces fueron los siguientes:

1. Las pasadas investigaciones sobre medios han puesto claramente de manifiesto que ningún medio enfatiza más el aprendizaje que otro, si no tenemos en cuenta otra serie de elementos como: las tareas de aprendizaje, los elementos simbólicos, el currículum, los contenidos o la estructuración de éstos,

2. la nueva tecnología es probable que enseñe mejor que su predecesora porque posee mejores materiales instruccionales y por la novedad,

3. las futuras investigaciones se realizarán en el contexto de las ciencias cognitivas; y,

4. no es cuestión de preguntarnos solamente cómo y por qué un medio opera en la instrucción y el aprendizaje, sino también por qué puede ser utilizado. (Cabero, 1991, p. 15)

Las conclusiones de Clark y Salamón marcan las principales limitaciones de la investigación previa e indican rutas o "nuevos caminos" para el avance científico en el campo de la tecnología educativa. Pese a ello, en la primera década del siglo XXI prevalecen visiones pragmáticas y funcionalistas de la enseñanza y, por ende, de los artefactos de mediación, porque superarlas requiere de investigación en las áreas deficitarias señaladas, que es justamente lo que aún no se hace. Elementos como el currículum, los contenidos y la 
estructuración de ellos en los planes de estudio, así como los contextos y los enfoques pedagógicos se deben investigar cuando se integra tecnología en los procesos de enseñanza y aprendizaje. Esta es, como se mencionó, una tarea pendiente.

\subsection{La investigación sobre tecnologías de la información aplicadas a la educación en la década de 1990: los enfoques emergentes}

La teoría de la comunicación, junto con la teoría de sistemas y las teorías del aprendizaje de las psicologías cognitiva, psicogenética y sociocultural son la base de una nueva concepción pedagógica de la Tecnología Educativa de finales de la década de 1980, que surgió como alternativa a las posiciones "fisicalista" y conductista que predominaron durante la primera mitad del siglo XX.

En la tradición conductista la clase y las actividades pedagógicas se centran en el docente, ya que es el encargado de desarrollar “... una adecuada serie de arreglos de contingencia de reforzamiento y control de estímulos para enseñar [mientras la actividad del alumno] se ve fuertemente restringida por los arreglos de contingencias del profesorprogramador" (Hernández, 1998, p. 94).

Por el contrario, en los paradigmas emergentes como el cognitivo, el construccionista, el sociocultural y el psicogenético, que ganan fuerza y adeptos en la comunidad científica a partir de la década de 1990, la clase se centra en el alumno y el profesorado desempeña el papel de mediador, organizador, promotor y agente cultural. Para esos enfoques la formación del profesorado en teoría de la enseñanza y el aprendizaje es fundamental para que efectivamente utilice las TIC en su práctica docente de forma innovadora.

En cuanto al cambio educativo y el uso innovador de las TIC en la enseñanza, en el paradigma cognitivo, cuyo principal exponente es David Ausubel, se propone que:

... el profesor parte de la idea de un alumno activo que aprende significativamente, que puede aprender a aprender y a pensar. El papel del docente, en este sentido, se centra especialmente en la confección y la organización de experiencias didácticas para lograr esos fines. Igualmente, su formación deberá orientarse en tal dirección. Las diferencias con el profesor "tradicionalista" consisten en que no debe centrarse exclusivamente en la enseñanza de la información, ni en intentar desempeñar el papel 
protagónico (es el que sabe, el que da la clase, etc.) en detrimento de la participación cognitiva de los alumnos (Hernández, 1998, p. 135).

En el paradigma psicogenético, desarrollado por Jean Piaget, se considera que:

... el maestro debe encaminar sus esfuerzos docentes a promover el desarrollo psicológico y la autonomía de los educandos. Desde esta perspectiva, el profesor, junto con el alumno y los saberes curriculares, es una parte importante de la relación tripolar existente en todo acto educativo. (Hernández, 1998, p. 197)

En el paradigma sociocultural, desarrollado por Lev Vygotsky,

El profesor debe ser entendido como un agente cultural que enseña en un contexto de prácticas y medios socioculturalmente determinados, y como un mediador esencial entre el saber sociocultural y los procesos de apropiación de los alumnos [...]. Así, a través de actividades conjuntas e interactivas, el docente procede promoviendo zonas de construcción para que el alumno se apropie de los saberes, gracias a sus aportes y ayudas estructurados en las actividades escolares, siguiendo cierta dirección intencionalmente determinada. (Hernández, 1998, p. 234)

En el cuadro 4, elaborado con base en la noción de paradigma de Kuhn (1980), se muestran las diferencias entre las teorías psicoeducativas conductista, cognitiva, psicogenética y sociocultural (Hernández, 1998).

La investigación desde el enfoque epistemológico constructivista, particularmente los paradigmas cognitivo, psicogenético y sociocultural, así como la investigación desde el enfoque sistémico, superan la perspectiva reduccionista inicial de la investigación en medios, para dar paso a nuevos problemas que involucran aspectos actitudinales del docente y del aprendiz, así como factores organizacionales y curriculares. Las principales conclusiones de esa tendencia son:

1. Las actitudes negativas [tanto de docentes como de estudiantes] hacia el ordenador influyen sobre el proceso de aprendizaje, desarrollando, por ejemplo, ansiedad hacia el medio informático y condicionando los resultados que se obtengan.

2. El trabajo con el ordenador puede desarrollar actitudes favorables hacia el mismo. 
3. Las actitudes de los alumnos hacia la informática son diferentes según el sexo y la clase social de los alumnos.

4. Las actitudes hacia los ordenadores se pueden ver favorecidas por el estudio de la informática.

5. La información de los alumnos, puede ser una técnica relevante para la evaluación de "software" informático educativo.

6. Las actitudes que los estudiantes tienen hacia el ordenador pueden verse marcadas por las actitudes que hacia el medio tengan sus profesores (Cabero, 1991, p. 15).

A partir de la investigación desde los enfoques teóricos emergentes se determinó que la perspectiva reduccionista de la tecnología aplicada a la educación ha sido poco exitosa en cuanto a capacidad explicativa, por lo que se propone una visión sistémica del fenómeno en la que se consideren todos los elementos que entran en juego para que la tecnología se utilice para favorecer el cambio y la innovación en la enseñanza y el aprendizaje. Ese enfoque respalda el ajuste de la educación a las demandas y expectativas educativas de la sociedad actual. Entre sus retos están el estudio del papel del profesorado en el cambio educativo y el análisis del uso de esas tecnologías en modelos de enseñanza alternativos al conductista y al tradicional (Bates, 2001; Zabalza, 2002, 2003). En cuanto al profesorado, la investigación muestra que el éxito de la enseñanza apoyada en tecnologías de la información, depende de las actitudes, juicios y creencias del profesorado sobre los medios.

Con base en un meta-análisis de la investigación sobre la integración de las TIC en la enseñanza hecho en los Estados Unidos en la década de 1990, por The Association for Educational Communications and Technology se determinó que los diez principales obstáculos para que esos medios se utilicen en forma apropiada e innovadora en educación son:

1. Falta de visión: Si entendemos "la visión" como la concepción de lo que es posible, no ha habido una visión apropiada del papel de la tecnología en educación, capaz de lograr que mejore la enseñanza y el aprendizaje.

2. Ausencia de planificación: la falta de visión conlleva a la falta de planeamiento, ya que no hay planes para capacitar al personal docente, ni para modificar el currículo o evaluar resultados. 
3. Apoyo insuficiente a la investigación en el campo: La investigación ha demostrado que, en la mayoría de los casos estudiados, los docentes realmente no sabían cómo utilizar estas tecnologías en la enseñanza y el aprendizaje, y no contaban con tiempo y condiciones para experimentar con ellas y documentar estas experiencias. Dar espacios para la capacitación y la experimentación educativa con estas tecnologías es fundamental para que el profesorado realmente tome el riesgo de implementar de forma innovadora las TIC en su trabajo de aula.

4. Infraestructura limitada, inadecuada o ambas: $\mathrm{Si}$ se desconocen las necesidades e intereses de los usuarios, la infraestructura informática, por lo general, se diseña e implementa con base en criterios técnicos y económicos, por lo que sus resultados finales son realmente pobres, nulos e, incluso, negativos.

5. Limitado acceso al equipo y a otras tecnologías: por las razones expuestas en el punto anterior, el profesorado encuentra que, si bien hay laboratorios y equipo disponible, el tiempo y recursos que le son efectivamente concedidos para su uso en clase es limitado, lo que lejos de ayudar y facilitar las tareas de aula se convierte en un problema imposible de resolver; como consecuencia, en la mayoría de los casos, la mejor opción para el docente es descontinuar su uso y regresar a las prácticas tradicionales.

6. Falta de incentivos: El profesorado, como se ha señalado en los puntos anteriores, lejos de encontrar en la tecnología un apoyo a su trabajo, lo que consigue son problemas nuevos para los que no tiene respuesta o solución adecuada de parte de la administración, frustración e, inclusive, incremento de trabajo con resultados académicos mínimos, nulos e, inclus o, negativos.

7. Falta de soporte técnico: Uno de los problemas críticos en cuanto al uso de la tecnología es el adecuado funcionamiento de los equipos y el software. Si el profesorado no tiene la garantía de que va a contar con ayuda oportuna en el momento en que se presente un problema, probablemente después de la primera mala experiencia desista de utilizarlos en sus clases.

8. Desarrollo profesional insuficiente del profesorado: Es común que el profesorado no tenga el conocimiento técnico y pedagógico necesario para utilizar en forma adecuada las TIC en su trabajo de aula. Si esta formación no se propicia por parte de las autoridades educativas, difícilmente los docentes tendrán la Volumen 9, Número 1, Año 2009, ISSN 1409-4703 
motivación e interés necesarios para explorar el uso de esas herramientas en sus cursos y actividades académicas. El desarrollo profesional del profesorado es una responsabilidad ineludible de quienes administran la educación, particularmente en lo que respecta al uso en el aula de las TIC, dada la complejidad que encierran estos dispositivos, tanto en el nivel educativo como en el técnico.

9. Requerimientos de capacitación tipo "just-in-time" (“justo a tiempo"): debido a que cada nivel educativo y cada disciplina o área de enseñanza tiene sus propias características pedagógicas, es necesario el diseño de planes de capacitación docente coherentes con las particularidades, necesidades e intereses de cada sector y nivel de enseñanza. Se debe monitorear en forma permanente la evolución y desarrollo de dichas necesidades e intereses, a fin de responder en forma oportuna a los requerimientos de formación que se vayan generando a lo largo del tiempo.

10. Falta de dinero: la falta de recursos financieros y humanos para atender las necesidades administrativas, de infraestructura, equipo y formación del profesorado, asociados al uso adecuado de las TIC en la enseñanza y el aprendizaje es uno de los principales problemas de la educación contemporánea. (Mehlinger \& Powers, 2000) 


\section{Cuadro 4}

Cuadro comparativo de los paradigmas conductista, cognitivo, psicogenético y sociocultural

\begin{tabular}{|c|c|c|c|c|}
\hline $\begin{array}{l}\text { Componentes } \\
\text { del paradigma }\end{array}$ & $\begin{array}{l}\text { Paradigma } \\
\text { conductista }\end{array}$ & Paradigma cognitivo & $\begin{array}{l}\text { Paradigmá } \\
\text { psicogenético }\end{array}$ & Paradigma sociocultural \\
\hline Problemática & $\begin{array}{l}\text { Estudio de la conducta } \\
\text { observable. }\end{array}$ & $\begin{array}{l}\text { Estudio de las } \\
\text { representaciones mentales. }\end{array}$ & $\begin{array}{lr}\text { Estudio de la } \\
\text { psicogénesis } \\
\text { conocimiento. }\end{array}$ & $\begin{array}{l}\text { Estudio sociocultural de la } \\
\text { conciencia. }\end{array}$ \\
\hline $\begin{array}{l}\text { Fundamentos } \\
\text { epistemológicos }\end{array}$ & Empirismo & Racionalismo & $\begin{array}{l}\text { Constructivismo, } \\
\text { interaccionismo } \\
\text { relativismo. }\end{array}$ & $\begin{array}{l}\text { Mediación instrumental y social } \\
\text { del conocimiento. }\end{array}$ \\
\hline $\begin{array}{l}\text { Supuestos } \\
\text { teóricos }\end{array}$ & \begin{tabular}{lr} 
Modelo & \multicolumn{2}{c}{ estímulo- } \\
respuesta $\quad$ y & el \\
determinismo & del \\
ambiente en la conducta.
\end{tabular} & $\begin{array}{l}\text { Modelos de procesamiento } \\
\text { de información y de } \\
\text { representación } \\
\text { conocimiento. }\end{array}$ & $\begin{array}{l}\text { Teorías de los estadios } \\
\text { y la equilibración. }\end{array}$ & $\begin{array}{l}\text { La zona de desarrollo } \\
\text { próximo. } \\
\text { Actividad instrumental } \\
\text { mediada. } \\
\text { Las relaciones } \\
\text { socioculturalmente } \\
\text { organizadas constituyen el } \\
\text { origen de las funciones } \\
\text { psicológicas superiores. }\end{array}$ \\
\hline $\begin{array}{l}\text { Prescripciones } \\
\text { metodológicas }\end{array}$ & $\begin{array}{l}\text { Metodología experimental } \\
\text { inductivista. }\end{array}$ & $\begin{array}{l}\text { Inferencia de los procesos y } \\
\text { representaciones } \\
\text { inobservables. }\end{array}$ & Método clínico-crítico & $\begin{array}{l}\text { Experimental evolutivo, análisis } \\
\text { genético comparativo y método } \\
\text { microgenético. }\end{array}$ \\
\hline $\begin{array}{l}\text { Proyecciones de } \\
\text { aplicación } \\
\text { en educación }\end{array}$ & $\begin{array}{l}\text { Arreglo adecuado de las } \\
\text { contingencias de } \\
\text { reforzamiento, con el fin } \\
\text { de promover con } \\
\text { eficiencia el aprendizaje. }\end{array}$ & $\begin{array}{l}\text { La educación es un proceso } \\
\text { sociocultural de transmisión } \\
\text { de saberes y contenidos } \\
\text { valorados culturalmente. }\end{array}$ & $\begin{array}{lr}\text { La actividad } & \text { auto- } \\
\text { estructurante } & \text { y } \\
\text { constructiva del } & \text { alumno } \\
\text { tiene } \quad \text { un } & \text { lugar } \\
\text { protagónico y central en } \\
\text { las } \\
\text { educativas. actividades }\end{array}$ & $\begin{array}{l}\text { Las instituciones educativas y } \\
\text { la formación escolar propician } \\
\text { el desarrollo de las funciones } \\
\text { psicológicas superiores. }\end{array}$ \\
\hline
\end{tabular}

Fuente: Hernández, G. (1998) 
La falta de investigación es un obstáculo para una integración de las tecnologías en la enseñanza y el aprendizaje, que permita la superación de una perspectiva ideológicopragmática, reduccionista y economicista de esos medios. La investigación también permitiría superar enfoques pedagógicos obsoletos, que ya no responden a las demandas educativas de la Sociedad del Conocimiento y la Información.

Debido a que la enseñanza es un acto consciente y con propósitos determinados, el profesorado asume una posición y toma decisiones sobre los medios que va a utilizar para propiciar el aprendizaje. La línea de investigación sobre el profesorado ha hecho descubrimientos importantes respecto del papel que juega el docente en el cambio y la innovación en educación, y de los factores que inciden en el rechazo o aceptación de las TIC como herramientas para mediar la enseñanza. No obstante, aún es un campo que requiere investigación para describir y explicar en su justa dimensión las implicaciones de la mediación pedagógica con tecnología, particularmente desde los enfoques emergentes de la educación que se fundamentan en las teorías de Piaget y Vigotsky.

\section{Conclusiones}

La incorporación de bs medios en los procesos de enseñanza y aprendizaje ha evolucionado desde sus inicios con una tendencia de corte conductista, directamente enfocada en el desarrollo de la instrucción programada, donde el control de los aprendizajes está en función de homogeneizar y predecir los comportamientos y las conductas esperadas del estudiantado. Para esta concepción, el apoyo en instrumentos, equipos y el diseño del material didáctico escrito son fundamentales para asegurar una educación plenamente controlada y sujeta a las condiciones preestablecidas por quienes diseñan las situaciones de aprendizaje.

La evolución de la investigación sobre las teorías de aprendizaje, los medios y los procesos de enseñanza permitió un salto cualitativo respecto de las limitaciones encontradas en la instrucción programada conductista, porque no permitía ampliar ni generar procesos de aprendizaje socioculturalmente situados, que redundaran en aprendizajes significativos. Conforme estas limitaciones se hicieron más evidentes creció el interés por una investigación educativa basada en nuevas teorías del aprendizaje de corte epistemológico cognitivo y constructivista, que se consolidaron en la década de 1990. Paralelamente, el auge de las 
tecnologías de la información y la comunicación aplicadas al quehacer educativo permitió avanzar en la construcción de ambientes de aprendizaje mediados por equipos computacionales y procesos de enseñanza y aprendizaje que favorecieran el aprendizaje significativo, la creatividad y la innovación, donde la persona que aprende explora y construye individual y socialmente su aprendizaje, mientras el profesorado asesora, guía y es parte del proceso de aprendizaje. Esta nueva perspectiva implica una ruptura con el enfoque de la enseñanza de tipo tradicional-transmisionista y conductista.

El concepto de tecnología educativa incluye las teorías de aprendizaje, los enfoques sistemáticos y el desarrollo de los medios de comunicación aplicados al quehacer educativo. Inicialmente, la investigación en educación, que en general se orientan al mejoramiento de las condiciones de enseñanza y aprendizaje para asegurar el cumplimiento de los objetivos educacionales planeados y a la asimilación de los contenidos por los estudiantes, generó muchas definiciones y concepciones acerca del uso de los medios en la enseñanza, mostrando una transición de una fase pre-científica especulativa a una científica, capaz de conformar comunidades de especialistas que impulsan el desarrollo de paradigmas emergentes en el campo de la educación.

La teoría y la investigación sobre la tecnología aplicada a la educación debe permitir la adecuación de diferentes medios a los contextos particulares y a los grupos sociales, independientemente de su edad, nivel educativo y experiencia de aprendizaje. Los hallazgos de investigación son una oportunidad real y válida ajusta de manera flexible y socialmente situada los avances tecnológicos en correspondencia con las posibilidades y recursos educativos de cada país.

Las concepciones acerca del campo de acción de la tecnología educativa comparten el uso de los medios de comunicación, la aplicación de teorías de aprendizaje y la apropiación de las tecnologías de la información y la comunicación para el mejoramiento de las prácticas educativas. No obstante, estos componentes son improductivos sin un proyecto educativo capaz de integrarlos a un enfoque pedagógico con una visión integral de persona. La formación educativa con tecnología tiene que estar acompañada de propósitos y valores social e individualmente relevantes, que responda a las demandas educativas del presente. De lo contrario, es una inversión inútil que puede convertirse en un arma de doble filo, que lejos de propiciar la innovación fortalezca las viejas y obsoletas prácticas de enseñanza. 
Es inconcebible la tecnología aplicada a la educación sin la mediación del personal docente. No es posible que los equipos y herramientas tecnológicas, por sí mismas, produzcan o promuevan procesos significativos de aprendizaje. El uso apropiado de la tecnología para la educación requiere del compromiso y la formación del personal docente en correspondencia con los requerimientos del presente, donde la administración de las instituciones educativas juega un papel crucial. La actualización permanente del profesorado es primordial para un uso oportuno de las tecnologías de la información y comunicación en los procesos de enseñanza y aprendizaje, pero ello depende de la visión que oriente la toma de decisiones de quienes administran la educación.

La integración de tecnología en los centros educativos debe trascender la instalación de equipos y programas computacionales, debido a que estos productos por sí solos no tienen impacto ni favorecen el mejoramiento del aprendizaje. Aunado a lo pedagógico, es necesario el diseño de procedimientos de uso, mantenimiento, incorporación y apoyo al currículo en forma permanente. En ese contexto, las preguntas relevantes respecto de la tecnología y sus aportes a los procesos de enseñanza y aprendizaje no son cómo o por qué, sino para qué pueden ser utilizadas. Esto es un asunto de vital importancia en la integración de cualquier tecnología en procesos educativos en la actualidad.

En relación con los obstáculos mencionados por The Association for Educational Communications and Technology, para que los medios se utilicen en forma apropiada en educación se considera que el principal impedimento, una vez instalados los equipos, es la falta de un proyecto educativo liderado desde la gestión de las organizaciones educativas, capaz de fomentar en el personal docente la innovación curricular, la formación permanente en el uso de las tecnologías de la información y la comunicación, la incorporación transversal en el currículo de las posibilidades que ofrecen Internet y ambientes multimedia y virtuales. Los avances en cuanto acceso y tecnologías especializadas para personas con necesidades educativas especiales es incuestionable, pero no son suficientes para el cambio educativo que demanda la sociedad contemporánea. La enseñanza con los medios y el uso de la tecnología para la educación requiere de un compromiso de toda la comunidad educativa: el personal directivo, el profesorado, la población estudiantil, las familias, el Estado, y las organizaciones privadas y públicas.

La tecnología educativa lejos de ser un "saber hacer mecánico" debe promover un "saber hacer mediado" por proyectos educativos inclusivos, tolerantes de la diversidad personal y Volumen 9, Número 1, Año 2009, ISSN 1409-4703 
cultural, para transformar la realidad y a las personas, únicas capaces de forjar ambientes socialmente equitativos y ecológicamente responsables.

Una línea de investigación educativa para valorar el uso apropiado de la tecnología educativa es la evaluación de los medios y procesos desarrollados en los contextos de aplicación. Se sugiere que esta debe ser constante y tener el propósito, entre otros, de monitorear y brindarle un seguimiento a los siguientes aspectos:

a. La pertinencia de los programas y aparatos tecnológicos respecto del contexto sociocultural y económico de la población donde se implementan.

b. Las mejoras y problemas que provocan los medios en la enseñanza y en el aprendizaje.

c. Los planes y proyectos de formación del personal docente para la apropiación de las TIC en sus labores cotidianas.

d. La incorporación en los planes y la gestión de las organizaciones educativas de actividades innovadoras con el uso de las TIC para su utilización curricular y el mejoramiento de las funciones administrativas.

e. La puesta en funcionamiento de planes de mantenimiento y actualización de los equipos y el software utilizado en los centros educativos.

f. La gestión y administración de fondos económicos que aseguren los recursos necesarios para la actualización de los equipos.

g. La concordancia de los programas computacionales con una teoría de aprendizaje forjadora de aprendizajes significativos.

Los enfoques teóricos y la investigación sobre medios de enseñanza desarrollados durante el siglo $\mathrm{XX}$ muestran que la educación requiere de tecnologías accesibles y pertinentes con un proyecto educativo inclusivo. El acceso a las TIC en los centros educativos debe ir acompañado de políticas educativas capaces de transformarse en realidades generadoras de ambientes de aprendizaje social y tecnológicamente mediados. 


\section{Referencias}

Bartolomé, Antonio. (2001). Concepción de la tecnología educativa a finales de los ochenta. [Documento WWW]. Recuperado: Antonio.Bartolome@doe.ub.es. (Consulta marzo, 2002)

Bates, William. (2001). ¿Cómo gestionar el cambio tecnológico? Estrategias para los responsables de centros universitarios. España: Gedisa.

Cabero, Julio. (1991). Líneas y tendencias de investigación en medios de enseñanza [Documento electrónico]. Recuperado en mayo 2003 de http://edutec.rediris.es/documentos/1991/4.htm.

Cabero, Julio. (1991). Innovación educativa [Documento WWW]. Recuperado en setiembre 2002 de www.doe.d5.ub.es/te/any97/ gallegoforce/\#cabero1991.

Cabero, Julio. (1992). Innovación educativa [Documento WWW]. Recuperado en setiembre 2002 de www.doe.d5.ub.es/te/any97/gallego force/\#cabero1992.

Cabero, Julio. (2001). Tecnología educativa. Diseño y utilización de medios en la enseñanza. Barcelona: Paidós.

Casanova, Ursula \& Berliner, David. (1997). La investigación educativa en Estados Unidos: El último cuarto de siglo. Revista de Educación, (312), 43-80.

Gallego, María Jesús. (1997). Cuestiones y polémicas en la investigación sobre medios de enseñanza [Documento WWW]. Recuperado en abril 2003 de www.mgallego@platon.ugr.es.

Hernández, Gerardo. (1998). Paradigmas en Psicología de la Educación. México: Paidós.

Kuhn, Thomas. (1980). La estructura de las revoluciones científicas. Madrid: Fondo de cultura económica.

Mehlinger, H. \& Powers, S. (2000). Technology \& Teacher Education. A Guide for Educators and Policymakers. U.S.A.: College Teaching Series.

Patiño, José Félix. (2002). Computador, cibernética e información. Colombia: Panamericana.

Zabalza, Miguel. (2002). La enseñaza universitaria. El escenario y sus protagonistas. Madrid: Narcea.

Zabalza, Miguel. (2003). Competencias docentes del profesorado universitario. Calidad y desarrollo profesional. Madrid: Narcea. 